\title{
DOA Estimation of Ultra Wideband Signals by using UWB MIMO Antenna
}

\author{
Mohammed H. Miry \\ Electrical Engineering Department \\ University of Technology
}

\author{
Ghaida A. AL-Suhail, Ph.D \\ Computer Engineering Department \\ College of Eng., Basra University
}

\begin{abstract}
There has been a flourishing prospect of UWB technology in recent years in both communication and other purposes like microwave imaging and radar applications. In the next generation of wireless communications, Multiple Input Multiple Output (MIMO) communication system will be a key technology to enhance the communication efficiency. The popular method for estimation the direction of arrival of sources impinging on an array of MIMO sensors is Multiple Signal Classification (MUSIC) method is a which is a problem of great interest in MIMO communication system. In this work, we proposed structure of UWB antenna based genetic algorithm and based on the design of single UWB antenna from previous stage, we form a 10x1 UWB MIMO array antennas to estimation direction of arrival for sources. The proposed UWB MIMO array antennas exhibit good UWB characteristics. In this work, we used MIMO array antennas for application MUSIC method to estimation UWB signals
\end{abstract}

\section{General Terms}

Wireless communication

\section{Keywords}

Ultra Wideband (UWB); Antenna; Multiple-Input-MultipleOutput (MIMO); Direction of Aarrival (DOA)

\section{INTRODUCTION}

Nowadays, wireless communication systems are becoming increasingly popular. However, the technologies for wireless communication still need to be improved further to satisfy the higher resolution and data rate requirements. That is why ultra wideband (UWB) communication systems covering from 3.1 $\mathrm{GHz}$ to $10.6 \mathrm{GHz}$ released by the FCC in 2002 [1-6] are currently under development. The idea of Multiple Input Multiple Output (MIMO) system has been recently become a hot research for its potential advantages. MIMO system has been proposed as a new system with various applications [7,8]. Direction of Arrival (DOA) estimation is a problem of considerable interest in MIMO array. Many methods like Capon and MUSIC $[9,10]$, have been developed, they use the data received at a sensor array to perform DOA estimation. Estimation the Direction of Arrival (DOA) of waveform, impinging on an array of sensors has long been of great research interest in a noisy environment using an array of $\mathrm{L}$ sensors [11]. The signals parameters are of interest are of spatial nature, require the cross-covariance information among the various sensors, which are the spatial covariance matrix. This covariance matrix $\mathrm{R}$ consists of a noisy covariance, and signal covariance matrixes. The covariance matrix is estimated from a finite number of samples of the data vector. In this paper MUSIC method is used as it offers better performance than others. Genetic Algorithm (GA) $[12,13]$ is a global probability search algorithm, which emulates the biological evolution process of Darwin's genetic selection and natural elimination. It possesses selfadaptability, global optimization, and implicit parallelism, manifesting the strong capability in solving problems [8]. In this paper, based on genetic algorithm, it is proposed to design ultra wideband antenna.

\section{SINGLE ANTENNA DESIGN}

The model is proposed for design UWB antenna based on genetic algorithm. In the model, based on genetic algorithm, the antenna dimensions are selected. Where, each chromosome is used to encode the antenna dimensions. The proposed model is simple to implement and computationally more efficient. The main procedure of the model is described as follow steps

Step1. Using binary encoding scheme for each weight encoded and randomly generates initial population in genetic algorithm.

Step2. The fitness (objective) function for genetic algorithm is defined as $\mathrm{S}_{11}$ is defined as

$$
\text { Fitness }=\min \left(\sum_{f=3 G H z}^{14 G \mathrm{Gz}}\left|S_{11}(f)\right|\right)
$$

Step3. The individual who have a high fitness would be to the next generation by genetic.

Step4. Crossover and mutation operation can be used to deal with the current population to produce the next generation of the population.

Step5.Then decoding the new population and calculate the error.

Step6. Repeat step2 to step5 Go to step 3 until obtaining determined fitness value

Step7. Construct the UWB antenna with the selected string.

Fig. (1) shows the geometry of the initial antenna which is fed by $50 \Omega$ microstrip line. The antenna is built on a FR-4 substrate with $1.6 \mathrm{~mm}$ thickness, relative permittivity of 4.4 and loss tangent of 0.02 . Seven parameters $\mathrm{L}_{2}, \mathrm{~L}_{3}, \mathrm{~L} 4, \mathrm{~L}_{6}, \mathrm{R}$, $\mathrm{W}_{3}$ and $\mathrm{W}_{6}$, are to be optimized to achieve the design goal of the UWB antenna. For simplicity, the parameters $\left[\mathrm{L}_{1}=\right.$ $3.18 \mathrm{~mm}, \mathrm{~L}_{5}=6 \mathrm{~mm}, \mathrm{~W}_{1}=8.9 \mathrm{~mm}, \mathrm{~W}_{2}=1 \mathrm{~mm}, \mathrm{~W}_{4}=5.75 \mathrm{~mm}$, $\left.\mathrm{W}_{5}=0.46 \mathrm{~mm}\right]$ are maintained constant during the optimization. 


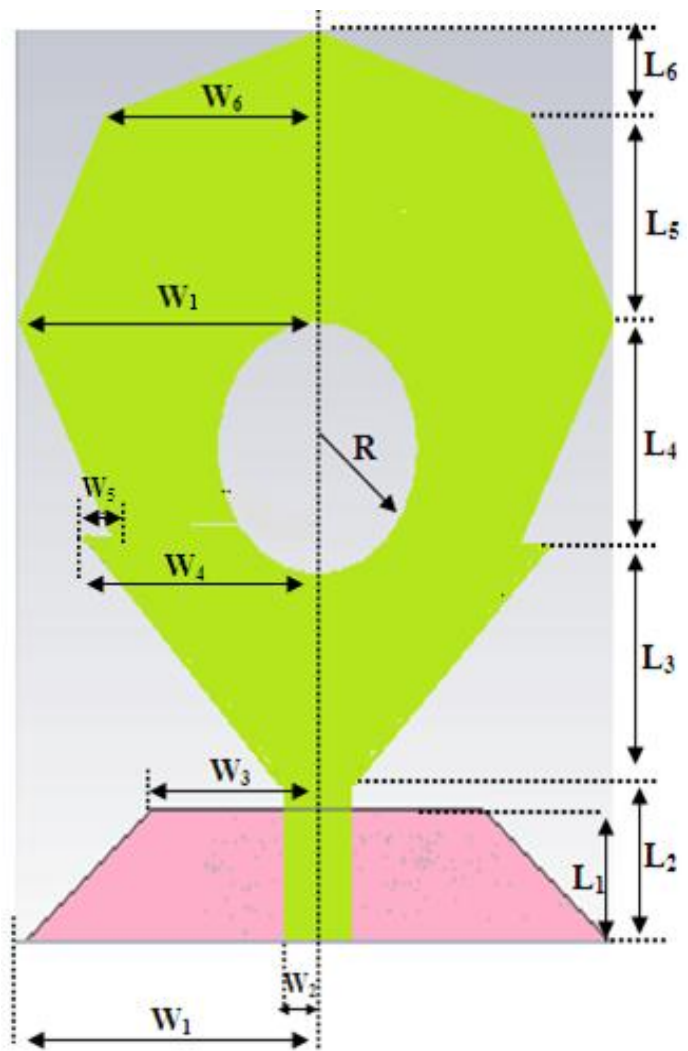

Top

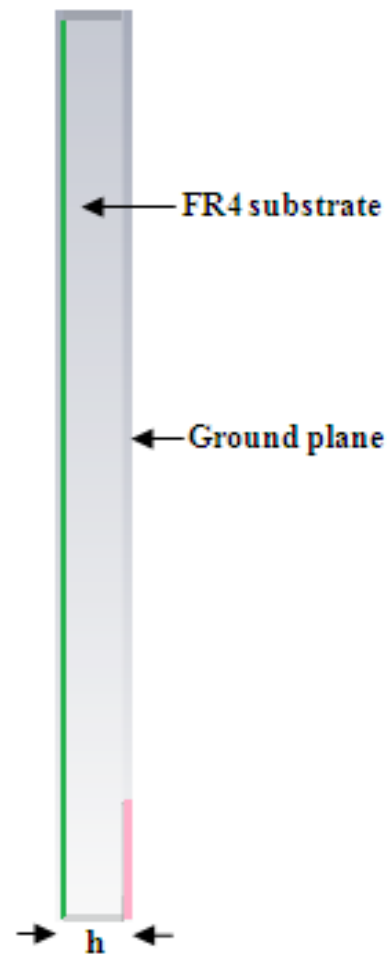

Side

Fig. 1: Geometries of the proposed antenna

\section{ANTENNA SIMULATION RESULTS}

In this section, full wave analyses of the proposed antennas in frequency domain are obtained by using the in CST Microwave Studio. In simulation, Parameter settings for the genetic algorithm are mutation rate $=0.06$ and population size $=20$. The antenna is optimized for its dimensions achieving the goal of reflection coefficient $S_{11}$ less than -10 $\mathrm{dB}$. The dimensions for proposed antenna obtained after optimization are $\left[\mathrm{L}_{2}=3.625 \mathrm{~mm}, \mathrm{~L}_{3}=5.755 \mathrm{~mm}, \mathrm{~L}_{4}=5.358 \mathrm{~mm}\right.$, $\mathrm{L}_{6}=0.866 \mathrm{~mm}, \quad \mathrm{R}=2.484 \mathrm{~mm}, \quad \mathrm{~W} 3=4.776 \mathrm{~mm}, \quad \mathrm{~W}_{6}=$ $5.544 \mathrm{~mm}]$. To verify the design and optimize antenna dimensions, numerical simulations have been used.

\subsection{Return Loss}

The desired frequency response is achieved as shown in Fig.2. It is apparent that the proposed antenna can cover the frequency band 3.37 to $13.54 \mathrm{GHz}$ for return loss less $-10 \mathrm{~dB}$. The size of the proposed UWB antenna is $21.6 \mathrm{~mm}$ by 17.8 $\mathrm{mm}$. This situation shows that the proposed approach can successfully optimize the application of a UWB antenna and find the optimal solution.

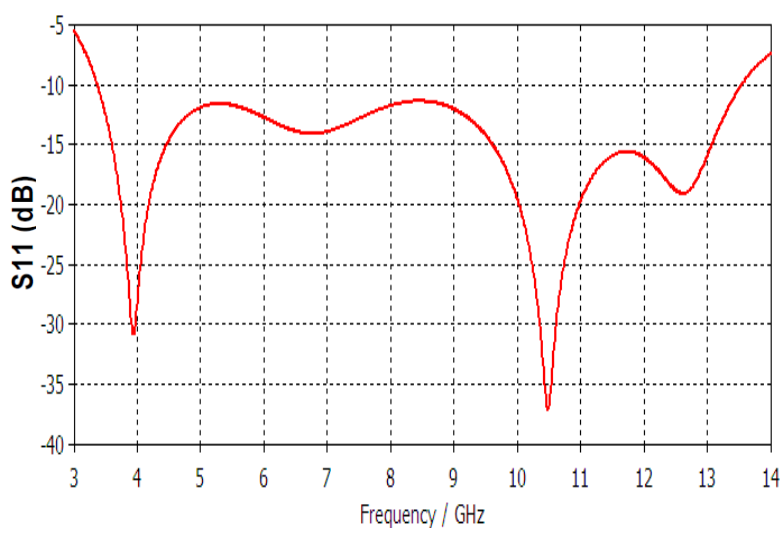

Fig. 2: The simulation reflection coefficient $\left(S_{11}\right)$ of optimum UWB antenna

\subsection{Radiation Pattern}

The 3D plot of simulated radiation patterns at specific frequencies about the antenna is illustrated in Fig. 3. The radiation patterns in the figure reveal that at frequencies they have nearly perfect omnidirectional radiation patterns. The proposed antenna has an acceptable quasi omnidirectional radiation pattern required to receive information signals from all directions. 


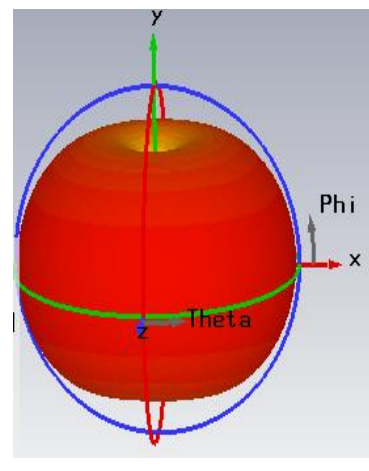

a

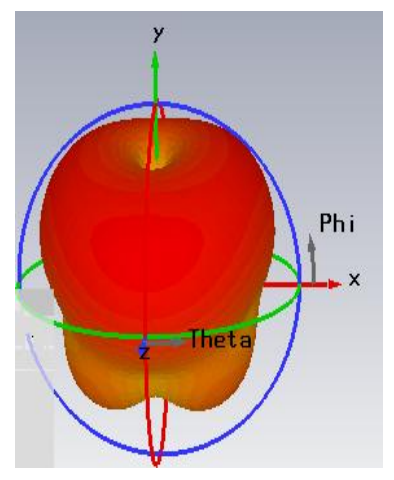

b
Fig. 3: Simulated 3D radiation patterns for proposed antenna at (a) $3.9 \mathrm{GHz}$, and (b) $10.5 \mathrm{GHz}$

\section{DESIGN UWB MIMO ARRAY ANTENNA TO ESTIMATION DIRECTION OF ARRIVAL IN WIRELESS COMMUNICATION}

In this section, based on the design of single UWB antenna from previous stage, we form a 10x1 UWB MIMO array antenna. The UWB MIMO array antennas are on same substrate, and they have symmetric structure. Figure (4) shows the geometry of the UWB MIMO antennas composed by ten identical radiating elements. The antenna separation is equal to one half of the wavelength corresponding to the highest frequency $\left(\mathrm{f}_{\mathrm{H}}=12.648 \mathrm{GHz}\right)$, Then, the distance between each antennas is equal $\mathrm{D}=11.85 \mathrm{~mm}$.

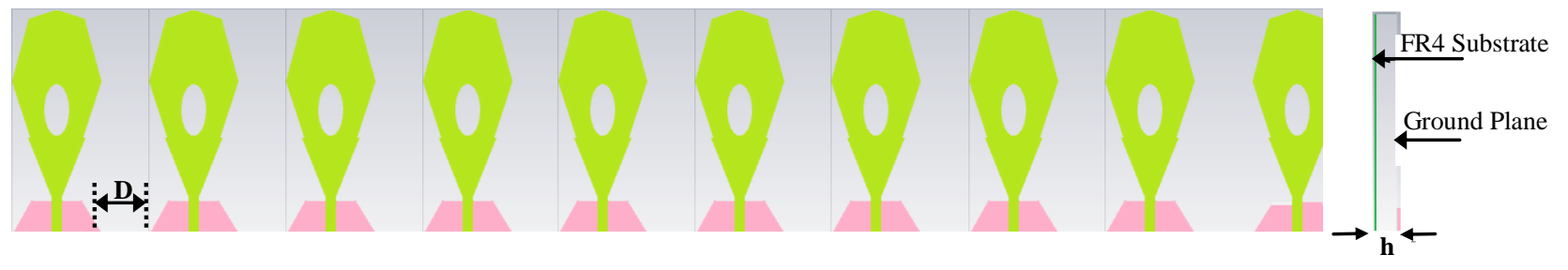

Fig. 4: The simulated geometry of the proposed 10x1 UWB MIMO Array antenna.

\section{MIMO SIGNAL MODEL}

Consider a uniform linear MIMO array of M sensors with inter sensor spacing of $d$. If different ultra wideband signals arrive at the array at angles $\theta_{1}, \theta_{2}, \ldots, \theta_{n}$, with respect to the array normal, the MIMO received signals data model is given by

$$
y(t)=\sum_{m=1}^{M} a\left(\theta_{m}\right) s_{m}(t)
$$

Where $s_{m}(t), \mathrm{m}=1,2, \ldots \ldots \ldots, \mathrm{M}$ denotes the ultra wideband signal and $a\left(\theta_{m}\right)$ is the steering vector of single signal at DOA $\theta_{m}$,The compact form of the output vector including an additive noise can be written as [14]

$\mathrm{y}=\mathrm{As}+\mathrm{n}$,

where $y=\left[\begin{array}{llll}y_{1}(t) & y_{2}(t) & \ldots . & y_{M}(t)\end{array}\right]^{T}$,

$n=\left[\begin{array}{llll}n_{1}(t) & n_{2}(t) & \ldots . & n_{M}(t)\end{array}\right]^{T}$ is zero-mean additive white complex Gaussian noise of variance $\sigma^{2}$. , and

$s=\left[\begin{array}{llll}s_{1}(t) & s_{2}(t) & \ldots & s_{n}(t)\end{array}\right]^{T}$

is the vector of source signals at time t. The steering-vector matrix A is given by [14],

$A=\left[\begin{array}{llll}a\left(\theta_{1}\right) & a\left(\theta_{2}\right) & \ldots & a\left(\theta_{n}\right)\end{array}\right]$,

Where $a\left(\theta_{l}\right)=\left[\begin{array}{lllll}1 & e^{j w_{l}} & e^{j 2 w_{l}} & \ldots & e^{j(M-1) w_{l}}\end{array}\right]^{T}$

and $w_{l}=k d \sin \left(\theta_{l}\right)$. It is assumed that the sources are mutually uncorrelated and that the auto-correlation of each source decays exponentially. It is also assumed that the signal and noise are uncorrelated.

The covariance matrix of the data vector would then be

$$
\begin{aligned}
& R=E\left[y y^{H}\right] \\
& =A E\left[s s^{H}\right\rfloor A^{H}+E\left\lfloor n n^{H}\right] \\
& =A P A^{H}+\sigma^{2} I,
\end{aligned}
$$

where $P=E\left[y y^{H}\right]=\operatorname{diag}\left[\sigma_{1}^{2}, \quad \sigma_{2}^{2}, \ldots, \quad \sigma_{n}^{2}\right]$, and $E\left[n n^{H}\right]=\sigma^{2} I$. Let $\mathrm{R}$ be eigen decomposed as [14]

$R=[U V]\left[\begin{array}{cc}\Lambda & 0 \\ 0 & \sigma^{2} I\end{array}\right]\left[\begin{array}{l}U^{H} \\ V^{H}\end{array}\right]$

where $\mathrm{U}$ and $\mathrm{V}$ are the signal subspace and noise subspace eigen vector matrices, respectively. It can be shown that $A^{H} V=0$ [15]. Or equivalently,

$$
a^{H}(\theta) V V^{H} a(\theta)=0
$$

at the true DOAs. An estimate of $\mathrm{R}$ is obtained from $\mathrm{N}$ samples of the data vectors as,

$$
\hat{R}=\frac{1}{N} \sum_{t=1}^{N} y(t) y^{H}(t) .
$$

If $\mathrm{R}$ is eigen decomposed as in eqn (6), one would arrive at the estimate of the noise subspace eigen vector matrix as $\hat{V}$. Since $\hat{V}$ is only an estimate, the left-hand side of eqn (7) 
would be minimum, and not zero, at the true DOAs if it is replaced by $\hat{V}$. Spectral MUSIC utilizes this fact, so that the ambiguity function [16]

$$
B(\theta)=\left(\frac{1}{a^{H}(\theta) \hat{V} V^{H} a(\theta)}\right)
$$

peaks at the true DOA

\section{Simulation and results}

We consider there are a two UWB signals impinging on UWB MIMO array antenna with signal-to-noise ratio (SNR) is fixed at $10 \mathrm{~dB}$ and the number of snapshots is 100 , the first signal is at $80^{\circ}$ and second signal is at $83^{\circ}$. In this case, the MUSIC search for the peak is illustrated in figure (5). From the figure (5), it is easily shown that two emitter signals is available due to the two peaks in the spectrums, it gave the result correct DOA for two emitter sources in direction $80^{\circ}$ and $83^{\circ}$.

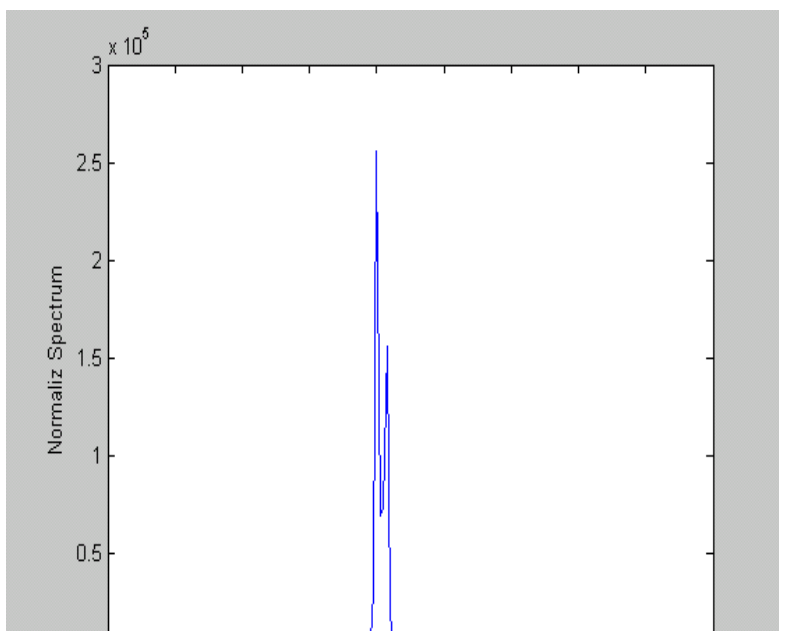

Fig. 5: DOA for Two Sources at $\theta_{1}=80^{\circ}$ and $\theta_{1}=83^{\circ}$ by using MUSIC Method

\section{CONCLUSIONS}

This paper is proposed model to design MIMO-UWB antenna used for estimation direction of arrival UWB signals. In this model, the geometry of the single UWB antenna body evolves through the optimization process to minimize antenna elements while maintaining a pretty return loss below a given specification based on genetic algorithm. In this work, based on the design of single UWB antenna from previous stage, we form a 10x1 UWB MIMO array antennas. In this work we estimation direction of arrival for source by using UWB MIMO array antennas

\section{REFERENCES}

[1] M. Koohestani and M. Naser-Moghadasi, "very compact ultra-wideband antenna with cpw-fed monopole", microwave and optical technology letters, Vol. 54, No. 3, March 2012

[2] S. M. Naveen, R. M. Vani, P. V. Hunagund J, "Compact Wideband Rectangular Monopole Antenna for Wireless
Applications" Wireless Engineering and Technology, 2012, 3, 240-243

[3] J. Xu, D.-Y. Shen, G.-T.Wang, X.-H. Zhang, X.-P. Zhang and K.Wu, "A Small UWB Antenna with Dual Band-Notched Characteristics", International Journal of Antennas and Propagation, Volume 2012.

[4] Mohammad Akbari, Mohsen Koohestani, Changiz. Ghobadi, and Javad Nourinia," compact CPW-FED printed monopole antenna with superwideband performance", microwave and optical technology letters,Vol. 53, No. 7, July 2011

[5] Ziani Kerarti Djalal, Meriah Sidi Mouhamed, "New Diamond Antenna for Ultra Wideband Applications", IJCSI International Journal of Computer Science Issues, Vol. 9, Issue 4, No 1, July 2012

[6] Giorgos Tatsis, Vasilis Raptis, Panos Kostarakis, "Design and Measurements of Ultra-Wideband Antenna", Int. J. Communications, Network and System Sciences, 2010, 3, 116-118

[7] A A. M. Isa, G. Markarian , M.S. M. Isa , N.Z. Haron and M.S.I.M. Zin," Hybrid TOA Based MIMO and DOA-Based Beamforming for Location and Positioning in WiMAX Networks" Journal of Telecommunication, Electronic and Computer Engineering, Vol. 4 No. 2. Pp.11-20 July December 2012.

[8] W. Shi, J. Huang and C. He, "Fast Algorithm for Maximum Likelihood DOA Estimation in MIMO Array", Proceedings of the World Congress on Engineering and Computer Science 2011 Vol I, San Francisco, USA, October 19-21, 2011.

[9] C. Guolang and W. Xifa, "Genetic algorithm and Its application," Beijing, Posts and Telecommunications News P A.C. Chang and J.C. Hung "Combining GA and iterative searching DOA estimation for CDMA signal" Neural Comput \& Applic (2010) 19:1003-1011

[10] S. Jin, G. Liao and J. Li," Joint DOD and DOA estimation for bistatic MIMO radar" Signal Processing 89, pp. 244-251,2009

[11] P. Stoica and R. Moses, "Introduction to spectral analysis", Prentice Hall Inc., New Jersey, 1997.

[12] H. Shil and Y. Deng, "Application of An Improved Genetic Algorithms in Artificial Neural Networks" Proceedings of the 2009 International Symposium on Information Processing, Huangshan, P. R. China, pp. 263-266, August 21-23, 2009

[13] F.Zaman, I. M. Qureshi, J. A. Khan, and Z. U. Khan, "An Application of Artificial Intelligence for the Joint Estimation of Amplitude and Two-Dimensional Direction of Arrival of Far Field Sources Using 2-LShape

[14] R. Sathish and G. V. Anand ,"Wavelet Denoising for Plane Wave DOA Estimation By MUSIC", TENCON 2003. Conference on Convergent Technologies for the Asia-Pacific Region Vol1, pp. 104 - 108,2003

[15] P. Stoica and R. Moses, "Introduction to spectral analysis", Prentice Hall Inc., New Jersey, 1997.

[16] J.C. Chang," DOA Estimation for Local Scattered CDMA Signals by Particle Swarm Optimization", Sensors journal 12, pp.3228-3242; 2012 